\title{
Evaluation of Ficus religiosa in Comparison to Captopril against Experimental Cardiotoxicity due to 5-Fluorouracil in Rats
}

\section{Ghadigaonkar Sushma ${ }^{1 *}$, Reddy Gopala A $^{2}$, B Kala Kumar ${ }^{3}$, M Lakshman ${ }^{4}$, Shiva Kumar ${ }^{2}$ and Anudeep Reddy ${ }^{1}$}

${ }^{1}$ Department of Veterinary Pharmacology and Toxicology, College of Veterinary

Science, Rajendranagar, Hyderabad, India

${ }^{2}$ PVNR, Telangana Veterinary University, Rajendranagar, Hyderabad, India

${ }^{3}$ Department of Veterinary Pharmacology and Toxicology, College of Veterinary

Science, Rajendranagar, Hyderabad, India

${ }^{4}$ Department of Pathology, College of Veterinary Science, Rajendranagar,

Hyderabad, India

*Corresponding Author: Ghadigaonkar Sushma, Department of Veterinary

Pharmacology and Toxicology, Mumbai Veterinary College, Mumbai, India.
Received: August 12, 2021

Published: August 21, 2021

(C) All rights are reserved by Ghadigaonkar

Sushma., et al.

\section{Abstract}

The present study was conducted to evaluate the cardioprotective effect of Ficus religiosa against 5-FU induced toxicity in rats. A total of 36 healthy male wistar rats of uniform age and weights were divided into 6 groups of 6 animals each. Normal saline (I), Ficus religiosa extract (II), Captopril (III) groups were control while next 3 groups (IV, V, VI) were concurrently treated with 5-FU, 5-FU+ FRLE, FU + CA. respectively for 14 day lipid peroxidation level was significantly higher while superoxide dismutase activity reduces in 5FU exposed groups. GST activity was significantly lower in vehicle control, Ficus religiosa and Captopril alone groups, concurrent treatment FR with FU showed decreased in LPO level and improvement in SOD, GST, GPx activities comparable to control groups. Histopathology study showed dilatation of cardiac muscle fiber, focal atrophy of cardiomyocyte with severe interstitial edema, and dilated blood vessels in 5-FU group. control groups showed no notable abnormal histological changes compared to 5-FU toxic control group. Heart sections of rats treated with 5-FU + FRLE, and 5-FU + Captopril showed morphology similar to that of control and exhibited reduced damage to cardiac architecture with fewer areas of congestion along with vacuolization. However, there was congestion of blood capillaries, swelling around the nuclei and focal haemorrhage in between the myocardial bundle. TEM of left ventricular myocardium of 5-FU toxic control group confirmed the light microscopic findings and revealed irregular nuclear envelope, dilated myofibrils with degenerating nucleus, dilatation of nucleus, dilatation of sarcoplasmic reticulum, disorganized, electron-dense and bizarre shaped mitochondria and congestion of blood capillaries. 5-FU significantly altered all parameters in study while groups that received FRLE and CA along with 5FU revealed significant improvement in all the parameters and the values were comparable to control group. Thus, it can be concluded that 5-FU is an anticancer that can cause cytotoxic effect, administration of FRLE altered those effects due to its antioxidant effect.

Keywords: Ficus religiosa; 5-Fluororuacil; Myocardial Antioxidants; HP; TEM

Citation: Ghadigaonkar Sushma., et al. "Evaluation of Ficus religiosa in Comparison to Captopril against Experimental Cardiotoxicity due to 5-Fluorouracil in Rats". Acta Scientific Veterinary Sciences 3.9 (2021): 49-54. 


\section{Introduction}

Over the world, people have been using plants as medicine from time as far back as immemorial. It is estimated by WHO (World Health Organization), that $80 \%$ of the population, majority in developing countries, rely on plant-based medicines for primary health care [1]. Plants, the manufacture the important medicinal drugs and regarded as the living chemical factories of nature. Plants were investigated for their complimentary, supplementary and synergistic action for treatment and maintenance of health. Currently, plants are poorly maximized as sources of new drugs [2]. There are numerous methods to select plant materials to search for novel medicinal plants/active compounds. Ethno-pharmacological information on medicinal plants is frequently of considerable significance to find novel possible medicinal plants or novel methods to use an already recognized plant. It was projected that $74 \%$ of the pharmacologically active plant-derived components were gotten after investigation was carried on the ethno-medical uses of the plants [3]. Another significant method to discover new medicinal plants is the phylogenetic approach in which an amount of extremely related species of plants supposed to comprise related chemical compounds (chemotaxonomy), are screened for their biological effects (Cotton, 1996; Vuorela., et al. 2004). The cardioprotective effect of medicinal plants is due to the presence of various phytochemicals such as flavonoids, phenolic acid, diterpenes and alkaloids [4,5]. Among the plants used by most herbalists as traditional medicines is Ficus religiosa (peepal). The plant belongs to the family Moraceae and it is found in almost all tropical countries. All parts of this plant (leaves, roots, seeds or stem barks) are used to treat bacterial, parasitic and fungal infections. The parts of $F$ religiosa are used for variety of treatments such as for hookworm, stomach pains, snake bite, leprosy, fever, dysentery, general body swellings, abortion, wound dressing, gargling, abdominal oedema, sterility, constipation as well as in child delivery [6]. The medicinal plants possess antioxidant, anti-inflammatory and cardioprotective activity that prove useful in ameliorating cardiotoxicity. Since the research aims at exploring the use of naturally occurring antioxidants from plant sources, it's likely impact would be the reduction in oxidative stress and reduction of/reversal from cardiotoxicity to normal cardiac functional status.

\section{Material and Methods}

A total of 48 healthy male Wistar albino rats of uniform age and weights were procured for the study from GenTox Bio-services Pvt Ltd, Hyderabad. The rats were kept in cages as per the specifications of CPCSEA and given standard feed (Pelleted rat feed from NCLAS, NIN, Hyderabad) and water. An acclimatization period of 2 weeks was observed before the start of the experiment. The experimental protocol was approved by the Institutional Animal Ethical Committee, College of veterinary science, Rajendranagar, Hyderabad (IAEC 1/VPT/19).

A total of 36 animals were randomized into experimental and control groups. They were divided into 6 groups with 6 animals in each group. The experimental design is shown in table 1.

\begin{tabular}{|c|c|c|c|}
\hline Group & Treatment & Protocol & No. of animals \\
\hline 1 & Normal control & Normal saline ( $2 \mathrm{ml}$ per kg b wt orally) for 14 days & 6 \\
\hline 2 & FRLE control & $\begin{array}{l}\text { FRLE (400 mg in } 2 \mathrm{ml} \text { normal saline per kg b.wt.) once } \\
\text { daily by oral intubation for } 14 \text { days }\end{array}$ & 6 \\
\hline 3 & Captopril control & Captopril 20mg/kg 14 days & 6 \\
\hline 4 & 5-Fluorouracil toxic control & $\begin{array}{l}\text { Toxic control } 5 \text {-Fluorouracil } 20 \mathrm{mg} / \mathrm{kg} \text { for } 14 \text { days (The } \\
\text { animals first receive normal saline ( } 2 \mathrm{ml} / \mathrm{kg} \text { b.wt./day) by } \\
\text { oral intubation for } 9 \text { days, and subsequently received } 5 \text {-FU } \\
\text { (20 mg in } 2 \mathrm{ml} \text { normal saline per kg b.wt.) once daily by } \\
\text { intraperitoneal injection for additional } 5 \text { days. }\end{array}$ & 6 \\
\hline 5 & $5 F U+F R L E$ & $\begin{array}{l}\text { Toxic control } 5 \text {-Fluorouracil } 20 \mathrm{mg} / \mathrm{kg} \text { from day } 9 \text { to } 14 \\
\text { + FRLE ( } 400 \mathrm{mg} \text { in } 2 \mathrm{ml} \text { normal saline per kg b.wt.) once } \\
\text { daily by oral intubation for } 14 \text { days }\end{array}$ & 6 \\
\hline 6 & 5FU + Captopril & $\begin{array}{c}\text { Toxic control 5-Fluorouracil 20mg/kg from day } 9 \text { to } 14 \\
\text { +Captopril } 20 \mathrm{mg} / \mathrm{kg} 14 \text { days }\end{array}$ & 6 \\
\hline
\end{tabular}

Table 1

Citation: Ghadigaonkar Sushma., et al. "Evaluation of Ficus religiosa in Comparison to Captopril against Experimental Cardiotoxicity due to 5-Fluorouracil in Rats". Acta Scientific Veterinary Sciences 3.9 (2021): 49-54. 


\section{Blood collection}

At the end of the experimental period, all the rats were anesthetized with light anesthetic ether and $2 \mathrm{ml}$ of blood was collected from the retro-orbital plexus, the serum was separated and used for the determination of diagnostic marker enzymes like LDH, CKMB, cTn, CRP, TG, TC, LDL, HDL and VLDL. It was also planned to estimate MDA, GSH, CAT, GPx and SOD. The histopathology and TEM of heart was also studied.

The thickness of the left ventricle wall

Each rat was anesthetized with ketamine-Xylazine and then thoracotomies. The beating heart was collected from the chest cavity and immersed washed three times with Tyrode's solution at room temperature to wash out blood. Small portion of heart was then immersed in ice-cold glutaraldehyde (2\%)-paraformaldehyde (2\%) fixative and fixed for at least $24 \mathrm{~h}$. Heart was removed from the fixative and trimmed off excessive fat and separate atria from the ventricles. Taken the weight and height of the left ventricle. The left ventricle was then serially cut into two halves. Measured the thickness of the left ventricular free wall using a Vernier calipers with a sensitivity of 0.01 [7]:

- Antioxidant Profile in Heart: Immediately after the sacrifice of animals, the heart tissue samples were collected and washed with ice-cold normal saline, blotted dry and stored at $-20^{\circ} \mathrm{C}$ for further analysis. From each sample, a piece of weighing about $500 \mathrm{mg}$ was taken and homogenized by tissue homogenizer (Heidolph, Germany) at $4^{\circ} \mathrm{C}$ and $10 \% \mathrm{w} / \mathrm{v}$ homogenate was prepared in cold PBS ( $\mathrm{pH}$ 7.4). Centrifuged the tissue homogenate at $2500 \mathrm{rpm}$ for $30 \mathrm{~min}$ and collected resultant supernatant. The total protein, reduced glutathione (GSH), superoxide dismutase (SOD), Catalase (CAT) and glutathione peroxidase (GPx) were estimated using the supernatants.

- Histopathology: The heart tissues were collected from the rats that were sacrificed at the end and fixed in $10 \%$ neutral buffered formalin (NBF) for histopathological studies. The fixed tissues were then processed and stained with Haematoxylin and Eosin (H and E) stain [8].

- Transmission electron microscopy (TEM) of heart: Small samples of heart were transferred to vials and fixed using $3 \%$ glutaraldehyde in $0.05 \mathrm{M}$ phosphate buffer $(\mathrm{pH} 7.2$ ) for $24 \mathrm{~h}$ at $4^{\circ} \mathrm{C}$ and post fixed using $2 \%$ aqueous osmium tetroxide in the same buffer for $1 \mathrm{hr}$. Samples were dehydrated in a series of graded alcohol and infiltrated and embedded in Araldite 6005 resin after the post-fixation. A glass knife was used to cut ultra thin sections (50 - $70 \mathrm{~mm}$ thickness) on a Leica Ultra cut UCT-GA-D/E-1/00 ultramicrotome and mounted on grids. Sections were stained with saturated aqueous uranyl acetate and then counter stained with $4 \%$ lead citrate [9] and were observed at various magnifications under a transmission electron microscope (Model Hitachi, H-7500) at RUSKA Lab, College of Veterinary Science, Rajendranagar, Hyderabad, India.

\section{Results and Discussion}

The richest bio-resources of drugs for traditional systems of medicine, pharmaceuticals, food supplements and chemical entities for synthetic drugs are the medicinal plants [10]. The biological activities of medicinal plants are due to the presence of various biologically active compounds like flavonoids, phenolic compounds, tannins, alkaloids, saponins, glycosides and steroids [4,5] and have been researched extensively for their therapeutic benefits in the healing of number of disorders.

Administration of 5-FU to rats was found to affect the thickness of the ventricle wall in this study. The increased thickness of ventricle wall by 5 -FU was successfully reduced and brought back to normal with FRLE, and Captopril treatment and presented in table 2.

\begin{tabular}{|l|c|}
\hline Group & Thickness of left ventricle wall (mm) \\
\hline 1. Normal control & $2.43 \pm 0.13$ \\
\hline 2. FRLE control & $2.71^{\mathrm{b}} \pm 0.11$ \\
\hline 3. Captopril control & $2.65 \pm 0.84$ \\
\hline 4. 5- FU toxic control & $4.46^{\mathrm{a}} \pm 0.15$ \\
\hline 5. 5-FU \pm FRLE & $2.60^{\mathrm{c}} \pm 0.08$ \\
\hline 6. 5-FU \pm Captopril & $2.81 \pm 0.04$ \\
\hline
\end{tabular}

Table 2: Thickness of left ventricular wall in different groups of rats.

Data are presented as Mean \pm SE; $n=6$; One way ANOVA; $(\mathrm{a}=\mathrm{p}<$ $0.05, b=p<0.001$ ) all group compared to the control group; $c=p$ $<0.05, \mathrm{~d}=\mathrm{p}<0.01) 5-\mathrm{FU} \pm \mathrm{FRLE}, 5-\mathrm{FU} \pm$ CA group compared to the 5-FU group. 5-FU= 5- Fluorouracil: FRLE = Ficus religiosa leaf (aqueous) extract.

Antioxidant present in heart has a very important role in protecting the heart and in allowing normal cardiac contractile per- 
formance. The results of myocardial antioxidants parameters in different groups of rats are given in table 3.
In the present study, the activity of SOD, CAT and GPx, and the concentration of GSH were decreased significantly in toxic control

\begin{tabular}{|l|c|c|c|c|c|}
\hline Group & $\begin{array}{c}\text { TBARs } \\
\text { (n mol/g wet } \\
\text { tissue) }\end{array}$ & $\begin{array}{c}\text { SOD } \\
\text { (mol/g wet tissue) }\end{array}$ & $\begin{array}{c}\text { CAT } \\
\text { (U/g tissue) }\end{array}$ & $\begin{array}{c}\text { GSH (mg/g wet } \\
\text { tissue) }\end{array}$ & $\begin{array}{c}\text { GPx } \\
\text { (U/g tissue) }\end{array}$ \\
\hline 1.Normal control & $80.99 \pm 0.94$ & $121.18 \pm 0.38$ & $22.01 \pm 0.94$ & $126.42 \pm 0.31$ & $34.12 \pm 0.52$ \\
\hline 2. FRLE control & $80.83 \pm 1.52$ & $105.44^{\mathrm{b}} \pm 2.02$ & $20.99^{\mathrm{b}} \pm 0.73$ & $119.15^{\mathrm{b}} \pm 0.52$ & $30.66^{\mathrm{b}} \pm 0.73$ \\
\hline 3. Captopril control & $62.65^{\mathrm{b}} \pm 1.85$ & $104.27 \pm 2.20$ & $20.62 \pm 0.31$ & $119.64 \pm 0.94$ & $30.56 \pm 1.78$ \\
\hline 4. 5- FU toxic control & $145.17^{\mathrm{a}} \pm 1.18$ & $77.89^{\mathrm{a}} \pm 2.20$ & $13.40^{\mathrm{a}} \pm 0.93$ & $65.07^{\mathrm{a}} \pm 0.31$ & $19.08^{\mathrm{a}} \pm 1.36$ \\
\hline 5. 5-FU \pm FRLE & $76.34^{\mathrm{b}} \pm 2.36$ & $122.35^{\mathrm{b}} \pm 1.35$ & $20.56^{\mathrm{b}} \pm 2.20$ & $129.34^{\mathrm{b}} \pm 1.15$ & $21.39^{\mathrm{b}} \pm 0.31$ \\
\hline 6. 5-FU \pm Captopril & $75.85^{\mathrm{b}} \pm 2.36$ & $119.38^{\mathrm{c}} \pm 1.52$ & $20.69 \pm 2.20$ & $120.36 \pm 0.94$ & $30.66^{\mathrm{c}} \pm 1.36$ \\
\hline
\end{tabular}

Table 3: Myocardial antioxidant profile in different groups of rats.

Data are presented as Mean \pm SE; $\mathrm{n}=6$; One way ANOVA; $(\mathrm{a}=\mathrm{p}<0.05, \mathrm{~b}=\mathrm{p}<0.001)$ all group compared to the control group; $\mathrm{c}=\mathrm{p}<0.05$, $\mathrm{d}=\mathrm{p}<0.01$ ) 5-FU \pm FRLE, 5-FU \pm CA group compared to the 5-FU group. 5-FU= 5- Fluorouracil: FRLE = Ficus religiosa leaf (aqueous) extract.
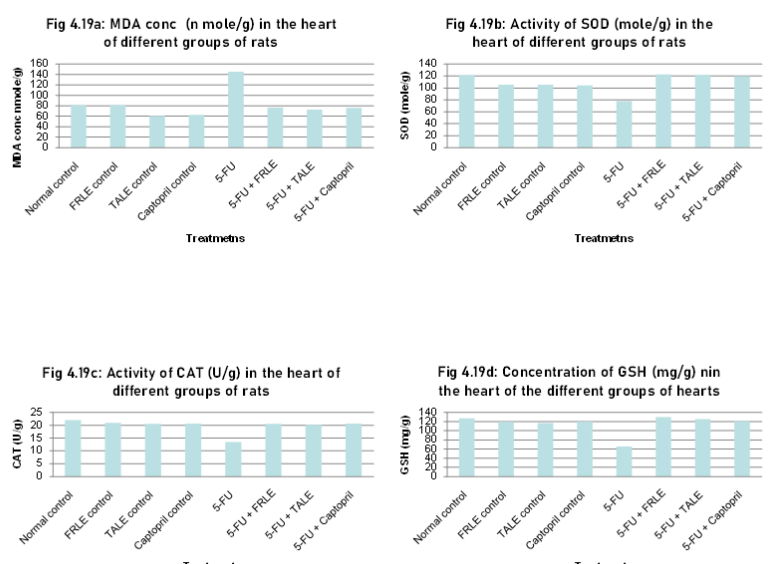

Figure 1

group 5FU. A decreased removal of superoxide anions, which can be harmful to the myocardium is responsible for decrease in SOD activity in heart. The activities of $\mathrm{H}_{2} \mathrm{O}_{2}$ scavengers viz., GSH, GPx and CAT were also decreased significantly after 5-FU treatment in the present study. Jaskanwal., et al. (2018) and Selmi., et al. (2018), who recorded significant reduction of SOD, CAT, GSH and GPX in guinea pigs treated with 5FU. Pre-treatment with FRLE, and Captopril prior to 5-FU effectively prevented increase in MDA and decrease in SOD, CAT, GSH and GPx, which may be correlated directly to the scavenging of radicals. Similar observations were recorded by Balakrishnan., et al. [11] and Devanesan., et al. [12] who observed that administration of aqueous extract of Ficus religiosa improves the SOD activity and decreased the Catalase activity due to less availability of NADPH.

The histopathology of left ventricle wall after the experimental period of 14 days is illustrated in figure $2 \mathrm{~A}$ to $2 \mathrm{G}$. Cardiac muscle fibers of the left ventricle sections showed normal architecture in the normal control group. Sections of cardiac muscle fiber of left ventricle from 5-FU control group showed dilatation of cardiac muscle fiber, focal atrophy of cardiomyocyte with severe interstitial edema, and dilated blood vessels, focal inflammatory, cell infiltration in between damaged myocardiocytes, atrophy, splitting disruption and hyalinised of cardiomyocyte in a focal manner was observed. Sections of cardiac muscle fiber of the left ventricle of all the treatment control groups showed no notable abnormal histological changes compared to 5-FU toxic control group. Similar observation were seen in Heart sections of rats treated with 5-FU + FRLE, and 5-FU + Captopril compare to normal control. Concurrent Administration of FRLE with 5-FU exhibited fewer areas of congestion along with vacuolization with reduced damage to cardiac architecture. How- 
ever, there was congestion of blood capillaries, swelling around the nuclei and focal haemorrhage in between the myocardial bundle, partial preservation of myofibrils and decreased breaks in muscle fibers, myofibrillar vacuolization and marked cellular infiltration.

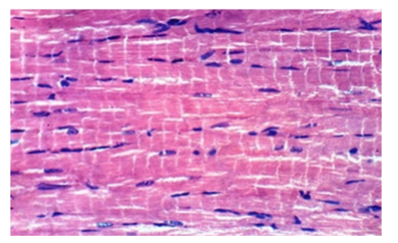

Figure A: Histological examination of section of heart of normal grou
(H \& E; X400)
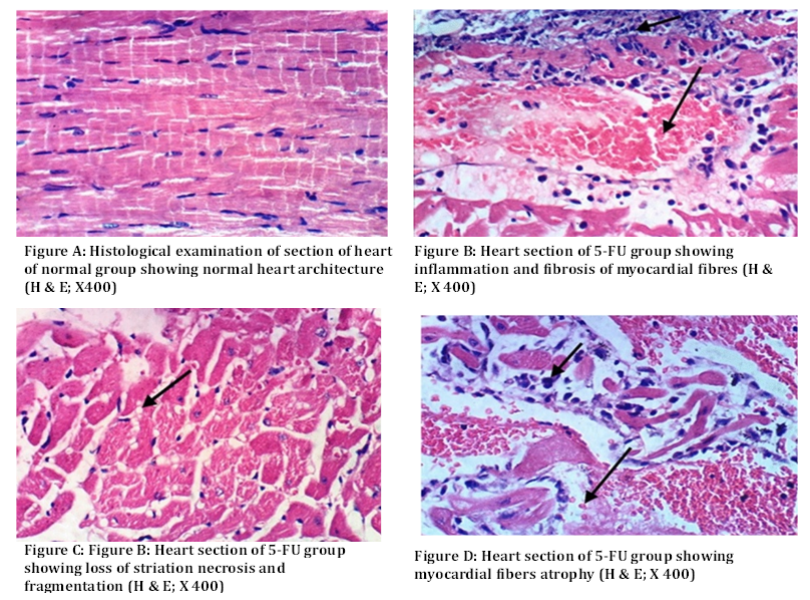

Figure B: Heart section of 5-FU group showing inflammation and fibrosis of myocardial fibres (H

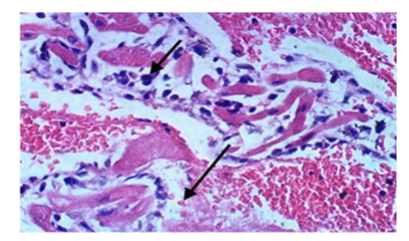
Figure D: Heart section of 5-FU group showing
myocardial fibers atrophy (H \& E; X 400)

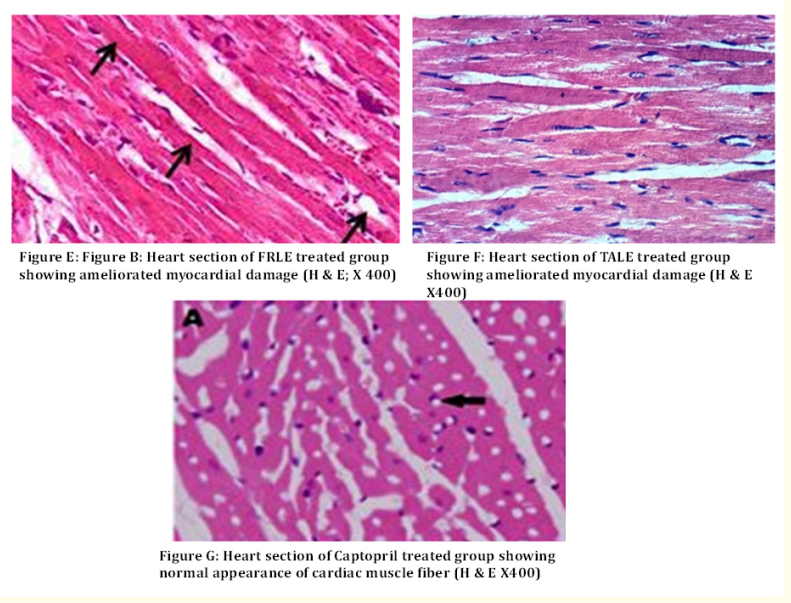

Figure 2: Histopathological studies.

The Ultrastructural findings on left ventricle wall after the experimental period of 14 days are illustrated in figure $3 \mathrm{~A}$ to $3 \mathrm{~F}$.

The normal structure of cardiac muscle fibers, which are connected end to end and side to side to one another by the intercalated discs, dilated myofibrils with normal nucleus and fibrin were observed in heart tissue of the control group after Ultrastructural examination. Around euchromatic nucleus, mitochondria

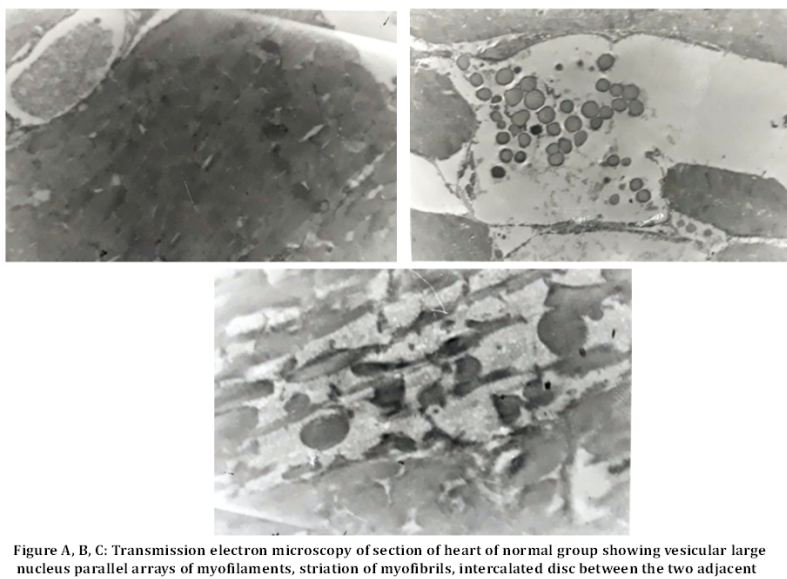
nucleus parallel arrays of my
cardiac muscle fibre $[\mathrm{X} 4000]$
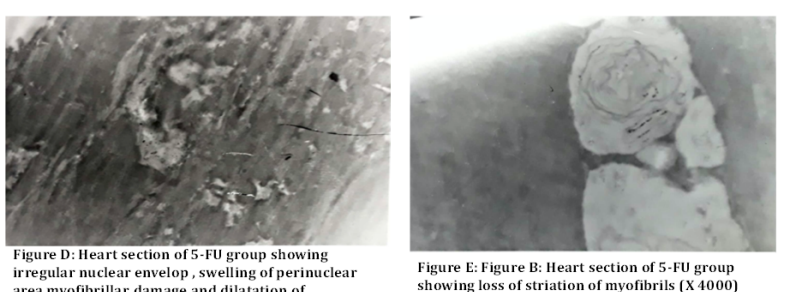

Figure E: Figure B: Heart section of 5-FU group

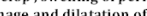
sarcoplasmic reticulum ( $X$ 4000)

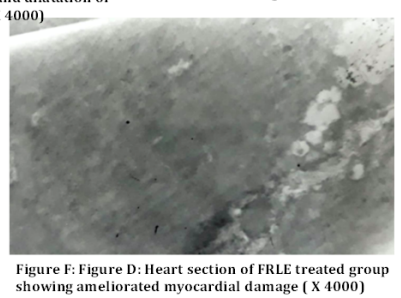

Figure 3: Transmission electron microscopy.

are arranged parallel to and in between the cardiac myofibrils. Ultrastructural examination of the left ventricular myocardium of FRLE, and Captopril treated groups confirmed the light microscopic findings and demonstrated dilated myofibrils with normal nucleus mild sarcoplasmic vacuolation and little myofibrillar damage. Ultrastructural examination of left ventricular myocardium of 5-FU toxic control group 5 confirmed the light microscopic findings and demonstrated irregular nuclear envelope, dilated myofibrils with degenerating nucleus, dilatation of nucleus, dilatation of sarcoplasmic reticulum, disorganized, electron-dense and bizarre shaped mitochondria and congestion of blood capillaries. Ultrastructural examination of the left ventricle of groups treated with 
5-FU + FRLE, and 5-FU + Captopril revealed myofilaments and nuclei appear somewhat normal. Degeneration of endothelial cells and undifferentiated myofibril looks like degenerated mass. The normal orientation of myofibril and architecture was found. Eman and Ghada [13] confirmed myofibrillar breaks and detachment of muscle strands accompanied by degeneration of muscle fibers due to 5-FU. Captopril is considered a standard drug for chemotherapyinduced cardiotoxicity. Hala [14] reported that pre-treatment with Captopril and its concomitant administration with 5-FU for 14days attenuated 5-FU induced myocardial damage and effectively reverted the abnormal structural changes near to normal [15].

\section{Conclusion}

In conclusion, the current study suggests that Ficus religiosa may has Therapeutic value in lowering cardio toxicity which, induced by 5 -Fluorouracil due to reducing serum lipid profile.

\section{Bibliography}

1. Korir RK., et al. "Antimicrobial Activity and Safety of two Medicinal Plants Traditionally used in Bomet district of Kenya". Research Journal of Medicinal Plants 6.5 (2012): 370-382.

2. Hostettman $\mathrm{K}$ and Terreaux C. "Search for new lead compounds from higher plants". Chimia 54 (2000): 652-657.

3. Farnsworth N R and Soejarto D D. "Global importance of medicinal plants”. In: Akerele, O., Heywood, V., Synge, H. (Eds.), Conservation of Medicinal Plants. Cambridge University Press, Cambridge (1991).

4. Barry Halliwell. "Antioxidants: The basic - what they are and how to evaluate them". Advances in Pharmacology 38 (1996): 2-20.

5. Ivanova D., et al. "Polyphenols and antioxidant capacity of Bulgarian medicinal plants". Journal of Ethnopharmacology 96 (2005): 145-150.

6. Chandrasekhar S B., et al. "Phytopharmacology of Ficus religiosa". Pharmacology Reviews 4 (2010): 195-199.

7. Charlers I M and Ogunde M. "Reversal of left ventricular hypertrophy by Propranolol in hypertensive rats". African Health Science 5 (2005): 29-32.
8. Singh U B and Sulochana S. "Handbook of histological and histochemical techniques". Premier publishing house, Hyderabad (1997): 1-63.

9. Bozzollo J J and Russel L D. "Electron Microscopy: Principle and technique foor biologists". Jones and Bartlett, Boston (1999): 670

10. Sukhdev S H., et al. "Extraction Technologies for Medicinal and Aromatic Plants". International Centre for Science and High Technology, Trieste (2008): 1-22.

11. Balakrishnan S., et al. "Protective effect of Ficus religiosa on Cyclophosphamide induced oxidative stress in brain". International Journal of Research in Pharmacy and Chemistry 4 (2014): 654-660.

12. Devanesan E B., et al. "Phytochemistry and Pharmacology of Ficus religiosa". Systemic Review Pharmacology 9 (2018): 4548.

13. Eman T M and Ghada M S. "Evaluation of cardioprotective activity of Leptidium sativum seed powder in albino rats treated with 5-Fluorouracil". Beni-Suef University of Basic and Applied Science 5 (2016): 208-215.

14. Hala F Abd-Ellah. "Ameliorative effect of Captopril against 5-Fluorouracil induced cardiotoxicity in rats: A study with light and electron microscopes". Journal of Applied Sciences Research 8 (2012): 863-872.

15. Mukherjee PK. "Alternative systems of medicine". In: Quality Control Herbal Drugs: An Approach to Evaluation of Botanicals, Business Horizons, Mukherjee, P.K. (Ed.). New Delhi, India (2002).

\section{Volume 3 Issue 9 September 2021} (C) All rights are reserved by Ghadigaonkar Sushma., et al. 